\title{
Using an Automated Air Traffic Simulation Capability for a Parametric Study in Traffic Flow Management
}

\author{
Heather Arneson* \\ NASA Ames Research Center, Moffett Field, CA, 94035 \\ Antony D. Evans ${ }^{\dagger}$ \\ Crown Consulting Inc., Moffett Field, CA, 94035 \\ Deepak Kulkarni, ${ }^{\ddagger}$ Paul Lee ${ }^{\S}$ \\ NASA Ames Research Center, Moffett Field, CA, 94035 \\ Jinhua $\mathrm{Li}^{* *}$ and \\ Universities Space Research Association, Moffett Field, CA, 94035 \\ Mei Yueh Wei ${ }^{\dagger}$ \\ NASA Ames Research Center, Moffett Field, CA, 94035
}

\begin{abstract}
Flight delays occur when demand for capacity-constrained airspace or airports exceeds predicted capacity. Demand for capacity-constrained airspace or airports can be controlled by a series of Traffic Management Initiatives (TMIs), which use departure and airborne delays, as well as pre-departure and airborne reroutes, to manage access to the constrained resources. Two systems exist in current and planned future operations to address imbalances between demand and capacity. The Collaborative Trajectory Options Program (CTOP) reduces demand to constrained resources by assigning strategic departure delay and predeparture reroutes. Reroutes are selected from Trajectory Options Sets (TOSs) submitted by airlines. As flights approach the constrained resource, the Time-Based Flow Management System (TBFM) is used to assign tactical delay to satisfy constraints. This paper describes experiments performed to study the impact of varying levels of airline participation in CTOP via submission of TOSs on ground delay and flight time, and the impact of departure uncertainty on TBFM delays. Results suggest that as CTOP participation increases, average ground delays decrease for all airlines, but to the greatest extent for airlines participating in CTOP. A threshold in CTOP participation, which varies with the constraint capacity, is identified beyond which there is relatively little further reduction in average ground delays. Similarly, given the likely level of CTOP participation, the capacity reduction for which CTOP would be an appropriate TMI is also identified. Results also suggest that high average departure errors and high variability in departure error can make the prioritization of TBFM internal departures in TBFM metering and scheduling infeasible. Departure errors at current levels are, however, acceptable.
\end{abstract}

\section{Introduction}

Zlight delays result when the capacity of critical resources, such as airspace or airports, is insufficient to meet

demand. Better matching between demand and available capacity can lead to efficiency improvements, with increased throughput, reduced delays, and more efficient flight trajectories. However, because of uncertainties in both demand and capacity (e.g., due to weather), such matching can be difficult. Mismatches in demand and capacity can be addressed by using a strategic pre-departure traffic flow management capability, such as the Collaborative

\footnotetext{
* Research Aerospace Engineer, Aviation Systems Division, M/S 210-6, Member.

$\uparrow$ Senior Scientist I, Aviation Systems Division, M/S 210-6, Senior Member.

* Aerospace Technologist, Intelligent Systems Division, M/S 269-2.

$\S$ Senior Research Associate, Human-Systems Integration Division, M/S 262-4.

${ }^{* *}$ Research Engineer, Aviation Systems Division, M/S 210-6, Senior Member.

$\dagger$ Computer Engineer, Intelligent Systems Division, M/S 269-1.
} 
Trajectory Options Program (CTOP), to pre-condition demand into the more tactical Time-Based Flow Management System (TBFM). This would enable TBFM to better manage delivery of demand to capacity constrained resources. CTOP adjusts demand in two ways. First, it can delay flights on the ground, reducing the arrival rate at the constraint resource. Second, it can reroute flights to avoid capacity constrained airspace or airports by selecting from a userspecified list of acceptable reroutes for each flight.

A wide variety of open questions exist addressing benefits of airline participation in CTOP and sensitivity of TBFM airborne delay to various sources of uncertainty. A number of human-in-the-loop (HITL) simulation studies have been carried out with experienced air traffic manager and controller participants ${ }^{1,2}$ to better understand the interaction of CTOP and TBFM. While these experiments have produced important results, the resources and time required to conduct real-time studies with human participants limits the number of HITL simulations that can be conducted and thus the number of experimental conditions that can be tested. In this paper, an automated simulation capability, ${ }^{3}$ called Traffic Management Initiative Automated Simulation (TMIAutoSim), is used to study the impact of airline participation in CTOP on ground delay and departure uncertainty on TBFM delay. TMIAutoSim can closely mimic the HITL simulation capabilities while automating both the human components and the collaboration between operational systems. ${ }^{3}$

CTOP and TBFM are described in more detail in Section II. In Section III, a variety of relevant open questions which need to be addressed are enumerated, followed by the specific objectives of this work. Section IV and Section $\mathrm{V}$ describe the two studies that were performed within the TMIAutoSim environment to meet these objectives. Section IV outlines a study that was conducted to quantify the impact of varying the levels of airline participation in CTOP on delay. Section V presents the results from a study used to quantify the impact of departure uncertainty on TBFM delays. Concluding remarks and notes for future work are given in Section VI.

\section{Background}

As described in Ref. 1 and Ref. 3, demand for capacity-constrained airspace or airports can be controlled by a series of Traffic Management Initiatives (TMIs), which use departure and airborne delays, as well as pre-departure and airborne reroutes, to manage access to the constrained resources. Two systems exist in current and planned future operations to address imbalances between demand and capacity, but in different operational contexts and different timeframes. One is more strategic in nature, with a planning horizon of hours and geographical reach that spans across the NAS. The second is more locally targeted to terminal airspace surrounding airports and has a shorter, more tactical time horizon. The capabilities comprising these two systems address different parts of the capacity management problem without close coordination.

Integrated Demand Management (IDM) is a NASA concept that aims to integrate and coordinate demand management across these systems to work together more effectively. ${ }^{1}$ Within the IDM concept framework, the strategic system may redistribute demand from an overloaded resource to one with reserve capacity, or allocate predeparture delay so that it is calibrated to variations in demand, and better matched to the demand that the tactical system can accommodate. This should allow the strategic system to manage delivery to the capacity-constrained destination more efficiently.

For strategic flow management, the IDM concept utilizes a new TMI called the CTOP. CTOP-specific capabilities support a characterization of the reduced capacity problem using multiple constraints, called Flow Constrained Areas (FCAs). CTOP can be initiated by the Air Traffic Control System Command Center when the capacity at a particular airport or region of airspace is expected to fall well below expected demand. When airspace is constrained, CTOP allows airlines to route their flights out of the associated FCA, or across a different FCA that has available capacity, by submitting a preference-weighted set of alternative routes called a Trajectory Option Set (TOS) from which the program can select. CTOP distributes traffic to reduce demand to manageable levels by delaying departures and allocating trajectories from airline TOSs to flights scheduled to pass through the specified FCAs. Departure delay is assigned via the Expect Departure Clearance Time (EDCT). The mechanism by which each alternative route is weighted is a Relative Trajectory Cost (RTC), in minutes, submitted by airlines, indicating the amount of ground delay the airline is willing to accept before preferring the next, lower ranked trajectory option. Trajectories and ground delays for each flight are calculated using the CTOP TOS allocation algorithm, using a ratio-by-schedule approach, and considering the RTC values assigned by each airline to each trajectory option. ${ }^{4,5}$

Although the objective of CTOP is to set departure times and routes such that maximum rates at capacity constrained resources are not exceeded, several factors impact a flight's actual arrival time at the resource, including departure error, winds, and changes in trajectory due to weather conditions. Thus, using only strategic tools, there is no guarantee that resource constraints will be satisfied. 
For tactical flow management, TBFM is an arrival scheduling tool that was developed to improve traffic delivery into capacity-constrained airports or airspace resources. ${ }^{1,6}$ As described in Ref. 3, TBFM is generally used to schedule flights arriving into a constrained airspace or airport. In this study, we focus on a specific example in which flights destined for Newark Liberty International Airport (EWR) are subject to arrival rate constraints at the runway threshold. TBFM creates a schedule for all inbound flights which provides a scheduled time of arrival for each flight at the runway threshold. Arrivals are sequenced by a "first-come first-served" scheduling algorithm according to their respective estimated arrival time at the runway threshold. A scheduled time of arrival is then assigned to each flight to satisfy the required inter-arrival spacing for a sequence of fixes through to the runway threshold. The arrival sequence and assigned scheduled time of arrival for each flight continues to change as the flight's estimated time of arrival changes, up until the flight crosses a specified TBFM freeze horizon, roughly $400 \mathrm{nmi}$ upstream of the runway threshold. At that point, the flight's scheduled time of arrival is "frozen" and the target arrival time at the runway threshold is fixed. Air traffic controllers are then responsible for managing aircraft to absorb delay in the air using delay vectors and speed clearances to meet these assigned scheduled times of arrival.

Operationally, there is a limit to the amount of airborne delay that can be absorbed within the TBFM freeze horizon. The problem becomes progressively more difficult as the amount of delay that must be absorbed increases. While this will depend on a variety of factors, such as distance from the TBFM freeze horizon to the runway threshold and airspace complexity, subject matter experts can be consulted to classify ranges of airborne delay for individual flights as acceptable, marginal, and unacceptable. Understanding the conditions under which airborne delay is pushed into the marginal or unacceptable realm is an open question that can be addressed by a parametric study.

Flights that depart from airports within the TBFM freeze horizon are referred to as "internal departures." These flights can receive delay on the ground in order to ensure that they fit into the overhead stream being controlled by TBFM. Their departure times are obtained using a TBFM departure scheduling function that assigns a departure time corresponding to an available arrival slot. Upon take off, flights can receive further airborne delay from TBFM, but this is typically small because the departure scheduling process ensures that these flights are departing into gaps in the overhead stream. Internal departure scheduling is performed in one of two ways. In the first scheduling paradigm, internal departures are given priority and are inserted into the overhead stream ahead of airborne flights. This may require assigning some delay to airborne flights in order to create a gap large enough to fit the internal departure. In the second, priority is given to airborne flights, with internal departures held on the ground until a large enough gap exists in the overhead stream. The prioritization of internal departures is a key enabler of the IDM concept, reducing the incidence of significant 'double delays,' in which internal departures receive high ground delays from CTOP, followed by high scheduling delays from TBFM because they cannot easily fit into the overhead stream. When internal departures are prioritized, this leads to an increase in airborne delay compared to using the scheduling paradigm in which airborne flights are prioritized. If the number of flights receiving marginal or unacceptable delay is too high when internal departures are prioritized, switching to the prioritization of airborne flights will reduce airborne delay in favor of greater ground delay for internal departures. ${ }^{3}$

Most of the software tools used in the HITL simulations were adopted, with some modification, for use by TMIAutoSim. Because the version of TBFM used in the HITL simulations is not suitable for use without human input, an alternative TBFM emulator was developed for TMIAutoSim based on an arrival scheduler developed for the Optimized Route Capability (ORC) ${ }^{7}$ tool. Functionality of the newly developed TBFM emulator including the implementation of the two TBFM scheduling paradigms described above (prioritizing airborne flights and prioritizing internal departures) and the automation of internal departure scheduling in accordance with TBFM procedures are described in detail in Ref. 3. An emulation of CTOP, called nCTOP (NASA CTOP) ${ }^{1}$ and constructed for the HITL simulations, was used to emulate the key functions of the operational CTOP system. nCTOP contains some added functionality from the original CTOP concept that may be introduced in the operational system in the future. The Multi-Aircraft Control System (MACS), ${ }^{8}$ a high-fidelity air traffic control simulation environment for prototyping scheduling systems and simulating air traffic, was used to simulate the air traffic. More information about the development and details of TMIAutoSim can be found in Ref. 3.

\section{Objectives}

A variety of parametric studies could be performed to improve understanding of the IDM concept and help researchers develop guidelines for use of the strategic and tactical tools under various conditions. These questions can be broken down into two categories: benefits of airline participation in CTOP and sensitivity of TBFM airborne delay to various sources of uncertainty.

While CTOP is an operational FAA TMI, first introduced in $2014,{ }^{4}$ it is still in limited operational use due to a variety of factors. For example, airlines need to do a significant amount of work to develop tools and procedures to 
build TOSs. Furthermore, this would impose an increased workload on airline dispatchers to review and approve trajectory options before submission into CTOP. This takes a considerable amount of research and development. Thus, airlines are coming online with CTOP capabilities at various rates. Some airlines with a greater capacity for research and development have been developing these tools while other carriers have not advanced as far. Quantifying the effects of varying levels of CTOP participation on ground delays and flight times serves two purposes. First, this analysis will provide an indication of potential benefits of CTOP participation to airlines. And second, the analysis will provide insights into the relationships between CTOP participation and the effectiveness of CTOP to reduce average ground delays to reasonable levels, given capacity constraints.

Sources of uncertainty in proposed IDM operations include: (1) flight departure error, when flights destined for the constrained airport do not take off exactly at their assigned departure time; (2) FCA capacity forecast error, when there is a mismatch between the predicted FCA rates used strategic planning with CTOP and the actual rates that are later used in tactical planning by TBFM; (3) airport arrival rate forecast error, a special case of FCA capacity forecast error when an FCA is located at the destination airport; and (4) the impact of weather and other traffic on flight time. Example research questions include identifying uncertainty values for each of these parameters at which the IDM concept is no longer effective, and whether they vary with the constraint capacity; whether or not the robustness of the concept can be improved by giving flights required times of arrival to meet at or upstream of the TBFM freeze horizon; and to what extent demand should be strategically set above forecast capacity in order to compensate for uncertainties, maintaining throughput.

From this list of parametric studies, this paper has two objectives:

1. Explore the impact of varying levels of airline participation in CTOP on ground delay, and

2. Explore the impact of departure uncertainty on TBFM delays.

These studies were chosen as they have been addressed to a limited extent on the HITL platform. The studies presented here are meant to augment the results found in those HITL studies and highlight the benefit of using TMIAutoSim in conjunction with HITL experiments to gain greater insight into the operational feasibility of the IDM concept.

\section{Sensitivity to Airline Participation in CTOP}

In this section, experiments are described that estimate the potential benefits, both in terms of overall system efficiency and airline/flight specific benefits of different levels of CTOP participation by airlines. Airlines are considered to be participating in CTOP if they provide TOSs with more than one trajectory option per flight. The potential benefits are studied with a limited number of HITL simulations in Ref. 9. In these HITL simulations, the portion of airline participation was varied from none up to $100 \%$ participation. The results indicate that nearly all of the overall system benefit can be achieved with only $50 \%$ CTOP participation. ${ }^{12}$ However, these HITL results are limited to the specific cases that were tested and their generalizability is unknown. Because TMIAutoSim does not require participant input during execution, a greater number of simulations can be run, allowing the analysis to be extended to generate statistically significant results which have improved generalizability. In this paper, benefits are estimated across a range of CTOP participation levels both for airlines that participate in CTOP and those that do not. However, the experiments presented also aim to explore the CTOP participation levels required to make a CTOP TMI effective at reducing average ground delay below an acceptable threshold, given the capacity constraint. Alternatively, given a CTOP participation level, these experiments aim to identify the capacity constraint values for which CTOP would be an effective choice of TMI.

\section{A. Experiment Details}

A common traffic scenario, describing origin airports, flight plans, and scheduled departure times for flights destined for Newark Liberty International Airport (EWR) was used for all results presented in the paper. This scenario consists of 195 flights, departing 66 origin airports. A total of 42 flights are airborne at the start of the simulation, of which 10 are international flights. All pre-departure flights are scheduled to depart within 4.5 hours of the start of the scenario. Flight times for flights that have not yet departed at the start of the simulation range from 30 minutes to over 4.5 hours. CTOP is used to schedule departures to meet arrival rate constraints at FCAs set at each of the three arrival fixes for EWR, as shown in Figure 1. A total of 39\% of flights are originally filed through the West fix, $24 \%$ through the North fix, and $37 \%$ through the South fix. The arrival rate constraints at these arrival fixes are varied. This experiment only involves the CTOP solution to the problem and thus MACS simulation of air traffic and simulated TBFM metering and scheduling were not required.

Airlines simulated to participate in CTOP specify a set of acceptable trajectories for each flight, in the form of a TOS, along with RTCs, which indicate airline trajectory preferences. TOSs were generated, as in Ref. 9, using a combination of inputs from subject matter experts, and clustering of 3 months of historical flight plan data from June 
to August 2015. Hierarchical clustering was used, with the dissimilarity between different routes described by Euclidean distance. ${ }^{10}$ Outlier detection was used prior to clustering to remove extreme routes, before the appropriate number of clusters was chosen by comparing average silhouette scores. ${ }^{11}$ Example TOSs are shown in Figure 2. All TOS routes went through one of the three meter fix FCAs. RTCs were calculated based on differences in flight time between each trajectory option, and the most preferred route, with an assumption of flight delay being 1.5 times the cost of ground delay, as in Ref. 12.

Airlines that do not participate in CTOP were still included in the program in that they were simulated to submit a single-option TOS (i.e., a TOS that consists of only their preferred route). Flights with a single-option TOS were only assigned ground delay by CTOP and were not rerouted.

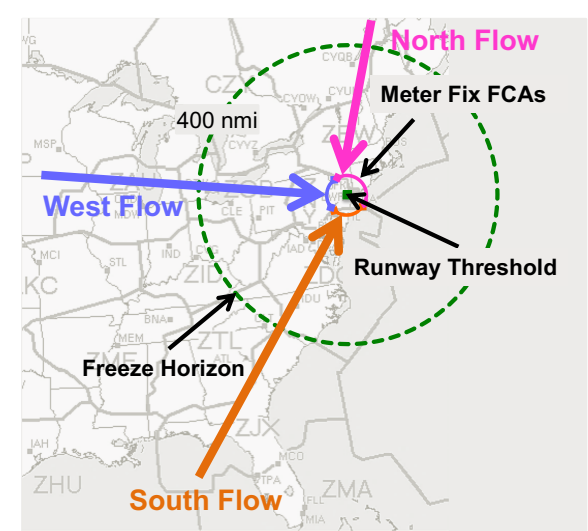

Figure 1. Location of FCAs at arrival meter fixes (North, South, and West) and/or at the EWR runway threshold and TBFM freeze horizon.

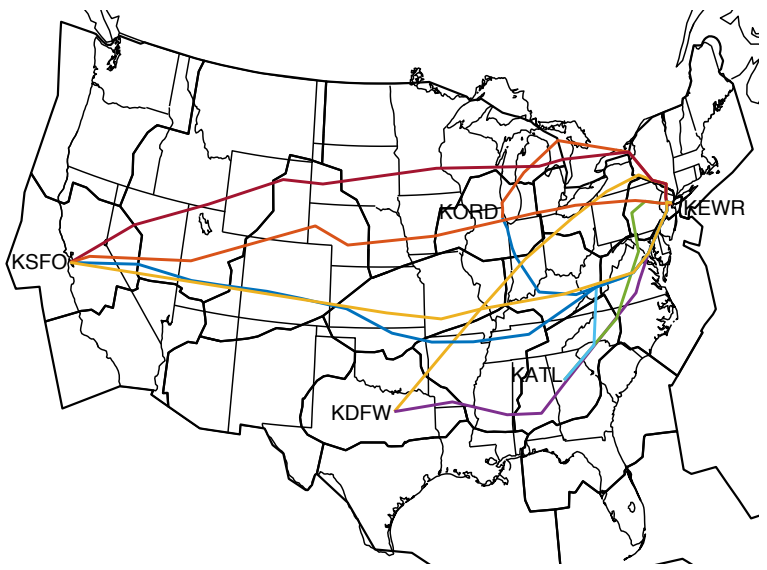

Figure 2. Example TOS, for flights from Chicago O'Hare (KORD), San Francisco (KSFO), Dallas-Fort Worth (KDFW), and Atlanta Hartsfield (KATL) International Airports, to Newark Liberty International Airport (KEWR).

The experimental setup used in these studies differ from that used in the HITL simulation presented in Refs. 12, and 9. As in Ref. 9, the North and South flows controlled by the arrival meter fix FCAs at the North and South arrival fixes, were set at clear-weather capacity ( 7 and 6 aircraft per quarter hour, respectively). However, the FCA flow rate for the West flow, controlled by the arrival meter fix FCA at the West arrival fix, was varied from 2 aircraft per quarter hour ( $40 \%$ of average demand) to a clear weather capacity of 5 aircraft per quarter hour ( $100 \%$ of average demand) (in Ref. 12 the FCA flow rate for the West flow is fixed at 3 aircraft per quarter hour). Furthermore, in contrast to Ref. 12, where an arrival rate of 11 aircraft per quarter hour was set for the arrival runway, the runway was left unconstrained here. This was done so that the effect of the constraints on the arrival fixes, and particularly the West Flow, could be isolated, without complicating impacts from the runway constraint. It is also important that only one flow (the West flow) is constrained, to ensure that trajectory options exist to other arrival fixes, on which there is available capacity. For each capacity constraint, nCTOP was run with CTOP participation varied from $1 \%$ to $72 \%$ (because some flights were considered exempt, TOS options were only calculated for $72 \%$ of flights). Benefits are measured using average delay for flights on the West flow, for aircraft submitting and not submitting TOSs.

\section{B. Results}

Figure 3 shows results for the case in which the West flow FCA capacity was limited to $60 \%$ of average demand, while the North and South flows were at clear-weather capacity. As the percentage of CTOP participation increases, average ground delays (blue curve) assigned to aircraft going through the FCA decreases. However, as the percentage CTOP participation increases to $40 \%$, further decrease in delay cost is minimal. The orange curve in Figure 3 shows the average RTC of aircraft that are routed out (i.e., given a trajectory option that is different from their preferred route, because the ground delay associated with their preferred route exceeded the specified RTC). This cost depends primarily on the RTCs associated with the alternate trajectories and is mostly independent of the percent CTOP participation. The difference between the blue and orange curves indicates the relative benefit, in terms delay minutes, of filing a TOS - airlines not filing a TOS would, on average, receive the ground delay shown (blue), whereas airlines filing a TOS would, on average, receive delay that is equivalent to the RTC delay cost (orange), because any ground delay greater than the RTC would result in the flight being routed out, incurring extra flight time of similar cost to the RTC. Figure 3 indicates that the benefit TOS filers have relative to non-TOS filers can be as much as 60 minutes, on average, in situations in which the percentage of TOS filers is small. This relative benefit decreases as the percentage 
of TOS filers increases. When the percentage of TOS filers is greater than $40 \%$, TOS filers as a group have only a small benefit over those not filing TOSs (in the order of 10 minutes), although there is still a benefit. Thus, as the percentage of CTOP participation increases, overall system performance improves while relative benefits to TOS filers declines.

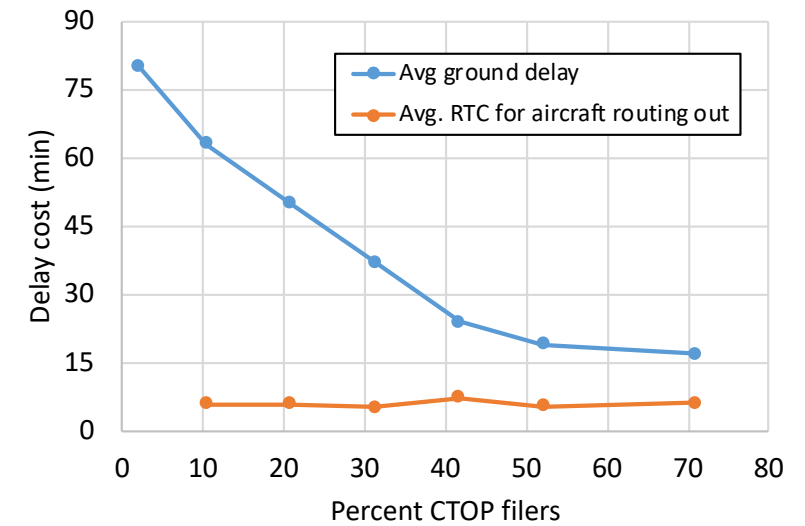

Figure 3. Delay cost as a function of CTOP participation, with West flow FCA capacity $60 \%$ of average demand (capacity deficit of $\mathbf{4 0 \%}$ ).

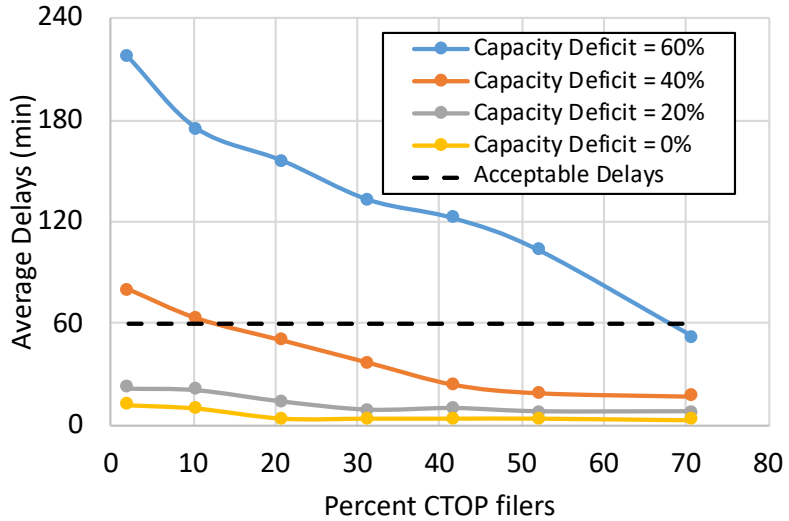

Figure 4. Average delay as a function of CTOP participation under various West flow FCA capacity constraints.

The percentage of CTOP participation at which delay cost plateaus for TOS filers was further investigated by varying the FCA capacity constraint applied to the West flow. We define capacity deficit to be the percentage difference between the available capacity and demand, i.e. capacity demand deficit is $100 \times\left(\frac{\text { demand-capacity }}{\text { demand }}\right)$. Figure 4 shows that the impact of CTOP participation on ground delays depends on the degree of capacity demand deficit. In all scenarios with non-zero capacity deficit, ground delays decrease as the percentage of CTOP participation increases. The larger the capacity deficit, the higher the CTOP participation beyond which there is no benefit to increased CTOP participation - at a $60 \%$ capacity deficit, there is still benefit to increasing CTOP participation, but at $0 \%$ or $20 \%$ capacity deficit, there is little benefit to increasing CTOP participation beyond $20 \%$ or $30 \%$, respectively. This is expected because after the percentage of CTOP participation is large enough to make up the capacity demand imbalance, further increase in the percentage of CTOP participation only results in a small decrease in average delays.

According to feedback from subject matter experts, if average West flow delay is over an hour, stakeholders are likely to consider alternative TMIs such as required advisory reroutes. By defining a 1-hour threshold of delays as acceptable, our analysis can be used to identify the minimum CTOP participation required for CTOP to be a suitable TMI option. As seen in Figure 4, what constitutes minimum required CTOP participation depends on the degree of capacity deficit. Given the likely level of CTOP participation, one can determine the type of capacity deficit scenarios in which CTOP use would be appropriate. Thus, in Figure 4, if acceptable delays are as indicated (1 hour), 30\% CTOP participation may mean that CTOP can be used effectively in scenarios with capacity deficit of $40 \%$ or less. These delays would depend on other factors such as duration of the CTOP TMI, as well as the specific scenario simulated, so further analysis is required to confirm this result.

\section{Departure Error Sensitivity}

Departure uncertainty is one of the major sources of uncertainty in the TBFM system. While the exact departure time affects the estimated arrival time at the TBFM freeze horizon, it also affects the order in which flights reach the freeze horizon and thus the order in which they are scheduled to reach the runway threshold. Slight changes in this order can lead to significant changes in delay assigned to particular aircraft. The time of departure for each flight is assigned by CTOP as an EDCT. For internal departures, a call-for-release time is then assigned by TBFM, delaying the flight beyond its EDCT. A flight is considered to be EDCT compliant if it departs within plus or minus five minutes of its EDCT, and call-for-release compliant if it departs between two minutes before and one minute after its call-forrelease time. However, in today's operations, many flights depart outside these compliance limits. ${ }^{12}$ In this section we examine the impact of departure error on assigned TBFM delay.

This study is divided into three parts. After describing some scenario details that are common across all parts, we look at the repeatability of results using TMIAutoSim in Section V-A. In section V-B we then examine the differences in TBFM delay assigned as the mean of the departure error shifts. Finally, we study the impact of changes in EDCT and call-for-release compliance variability on TBFM delay in Section V-C. 
While total delay is a good metric to understand the overall efficiency of the scheduling algorithms, operationally it is more important to understand how individual flight delays are distributed in the simulation. Air traffic controllers can relatively easily control flights to absorb a small amount of delay in the air, without turning off the TBFM system or putting flights into airborne holding. The problem becomes progressively more difficult as the amount of delay that must be absorbed increases. Based on subject matter expert feedback for the airspace simulated, TBFM airborne delay is considered operationally acceptable if it is below 7 minutes, marginal if it is between 7 and 14 minutes, and unacceptable if it is greater than 14 minutes.

The traffic scenario presented in Section IV-A is used for all experiments in this section. However, instead of FCAs at each meter fix, in these experiments a single FCA is applied at the runway threshold, with a rate of 11 aircraft per quarter hour, representing single runway operations on runway $22 \mathrm{~L}$. Given the initial scheduled departure times, the arrival rate at EWR would have been as shown in Figure 5, labeled 'Baseline'. EDCTs for pre-departure flights were calculated using nCTOP in order to meet the runway arrival rate constraint (labelled 'AAR' in Figure 5). Because some flights are airborne at the start of the simulation, and others are exempted from CTOP, the resulting rate (labelled 'CTOP' in Figure 5) does not meet the desired rate precisely. It is therefore necessary for TBFM to assign some amount of airborne delay in order to achieve the desired arrival rate at EWR. This is done by simulating flights in MACS, before using the

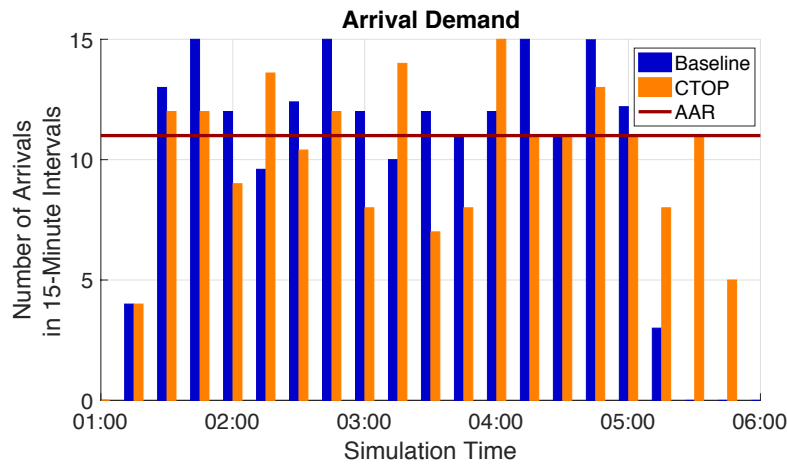

Figure 5. Baseline demand indicates arrival demand resulting from original schedule with no departure delay. CTOP demand indicates demand given CTOP EDCTs with no departure delay. Airport Arrival Rate (AAR) is the desired arrival rate.

TBFM emulator to simulate TBFM operations in post-processing. This applied additional inter-arrival spacing at the final approach fix of $0.4 \mathrm{nmi}$, which achieves as close to 11 aircraft per quarter hour as possible.

The TBFM freeze horizon was set at approximately $400 \mathrm{nmi}$ from the EWR runway threshold, as in current TBFM operations (shown in Figure 1). Aircraft departing from airports close to EWR (roughly within the freeze horizon) are considered internal departures and have their scheduled times of arrival at the runway threshold frozen as they depart.

\section{A. Simulation Repeatability}

As shown in Ref. 3, running the simulation multiple times with identical at real-time speed does not produce precisely identical results - total flight time varies by up to 5 seconds. In order to distinguish between the impact of departure error versus variations in simulation performance, experiments were performed to quantify the variations in TBFM delay caused by slight changes in simulation output over several executions of the scenario, with each run simulating exactly the same set of departure error values.

\section{Experiment Details}

The common traffic scenario was executed using the EDCTs generated by nCTOP. It was run five times with no departure error and five times with a fixed set of departure errors (i.e., the same departure errors were used in each of the 5 runs). This set of departure errors was extracted from empirical departure error distributions generated from observed departure errors from historical Ground Delay Programs and periods when TBFM internal departure scheduling was active, from June to August 2010. In these distributions, external flights had a mean departure error of 1.1 minute early and standard deviation of 14.5 minutes, while internal departures had a mean departure error of 0.6 minutes early, and standard deviation of 5.7 minutes. This set of departure errors has been used in previous work, ${ }^{1}$ $, 2,3,12$ and is used here for consistency. Both TBFM scheduling paradigms (i.e., prioritizing airborne flights and prioritizing internal departures) were applied in post processing on all ten of these simulation results.

\section{Results}

When the simulation is run using EDCTs from nCTOP and no departure error, TBFM must impose a total of nearly 2,000 minutes of delay (airborne and ground), on average across the 5 runs, in order to meet the runway arrival rate constraints. The introduction of departure error results in an increase in the amount of airborne delay required to meet runway arrival constraints. When airborne flights are prioritized, on average, 285 additional minutes of airborne delay are required across all flights when departure error is introduced (186 minutes when internal departures are prioritized). The standard deviations of total airborne delay assigned across all simulations for each scheduling paradigm, with and 
without departure error, ranges from 9.5 to 23.4 minutes. This indicates that errors due to simulation repeatability are small in comparison to total system delay differences caused by departure errors on the order of minutes.

TBFM airborne delay per flight for the paradigms in which priority is given to airborne flights and priority is given to internal departures is shown in Figure 6 and Figure 7, respectively. The bars indicate the average number of flights assigned a given delay value. The black lines at the top of the bar indicate the standard deviation of the number of flights assigned delay in that one-minute range over the five executions of the same scenario. Standard deviations are up to 1.2 aircraft when prioritizing airborne flights, and 0.6 aircraft when prioritizing internal departures. While not insignificant, the variability can be seen to be generally small in comparison to the numbers of flights plotted in each bin, indicating that the simulation repeatability is acceptable. Note that, while airborne delay increases when priority is given to internal departures, it remains below unacceptable levels and internal departure ground delay decreases significantly (shown in Refs. 1, 2 and 3) making it a key enabler of the IDM concept.

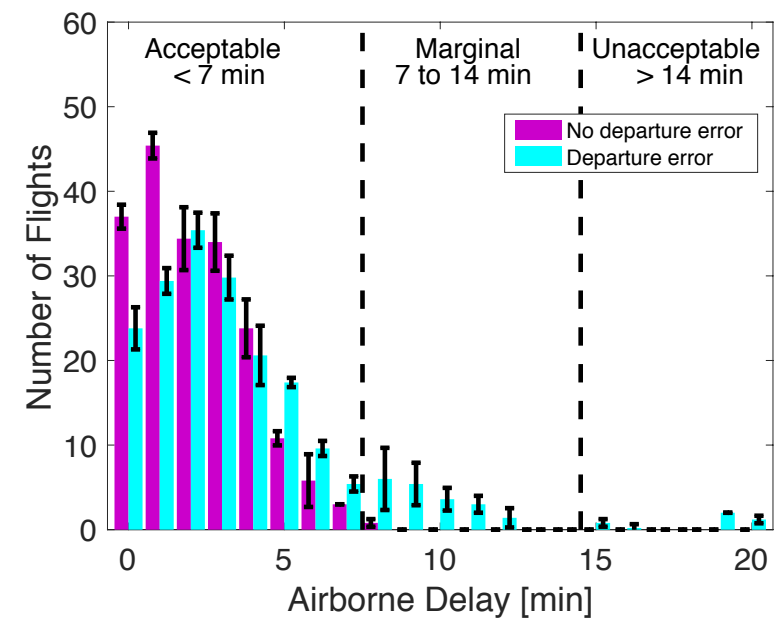

Figure 6. Priority given to airborne flights.

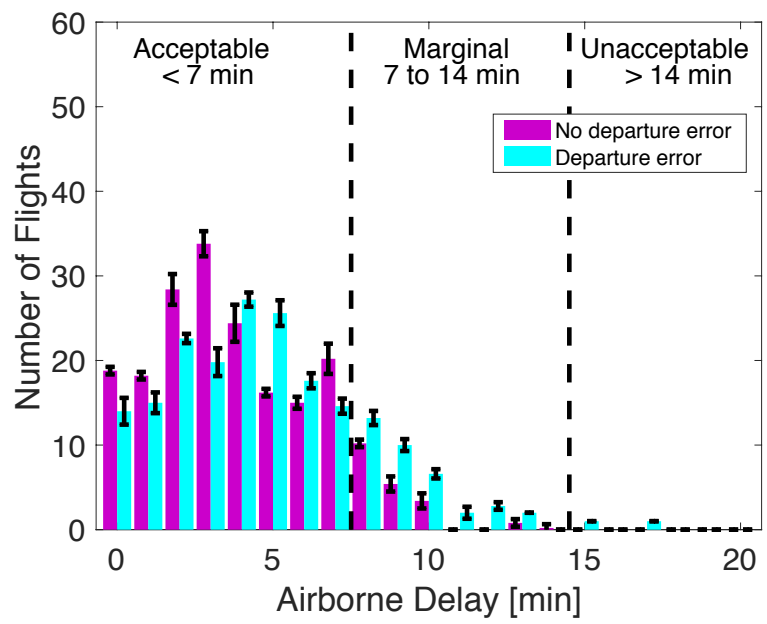

Figure 7. Priority given to internal departures.

\section{B. Impact of Departure Error Offset on TBFM Delay}

As demonstrated in Ref. 3 and shown in Figure 6 and Figure 7, a greater portion of assigned delay is categorized as marginal or unacceptable when internal departures are prioritized. Figure 6 and Figure 7 also show that, in both cases, delay is shifted from acceptable to marginal, and even unacceptable, when departure error is introduced. This indicates that, under the capacity constraints simulated, significant departure error may make the use of TBFM with priority given to internal departures, and even TBFM for arrival metering generally, impractical. The level of acceptable departure error that would allow feasible TBFM metering is considered in the following sections, first in terms of mean error (a fixed offset) in this section and then error variability in Section V-C.

\section{Experiment Details}

Five different experimental runs were completed to explore the impact of departure error mean on TBFM delay. The departures errors simulated were based on those used in previous work, ${ }^{1,2,3,9}$ but with the distribution mean shifted by a constant offset of $-10,-5,0,5$ and 10 minutes, for each of the 5 runs, respectively. After the offset constant was applied to the departure errors, the resulting departure errors for some flights were truncated to ensure that flights scheduled to depart early in the scenario would not depart before the start of the simulation. For the baseline distribution, with no shift to the mean departure error, $87 \%$ of flights were EDCT compliant with departure error within five minutes of the assigned EDCT. With a shift in departure error of $+/-5$ minutes, just over $58 \%$ of flights were EDCT compliant. Roughly $30 \%$ of flights were EDCT compliant after a departure error shift of $+/-10$ minutes. Both TBFM scheduling paradigms (i.e., prioritizing airborne flights and prioritizing internal departures) were applied in post-processing on all five of simulation outputs from MACS.

\section{Results}

The resulting required TBFM airborne delay for each run is shown in Figure 8 and Figure 9. As the mean of the departure errors is shifted away from zero, marginal and unacceptable delays increase, as expected. The data labeled "No error" and "Error with 0 min offset" are results from the repeatability study for the cases in which no departure error is injected into the scenario, and in which a fixed set of departure errors is injected into the scenario, respectively. 
Looking at the results for the TBFM scheduling paradigm that prioritizes internal departures in Figure 9, the number of flights assigned marginal delay is quite high, even at $+/-5$ minutes shift in departure error (i.e., 2.3 times and 1.8 times the number of flights given marginal offset when no departure error was assigned, respectively). At $+/-10$ minutes shift in departure error, unacceptable delay spikes to 16 and 33 flights, respectively. In an operational setting, controllers would likely discontinue the use of TBFM under the scheduling paradigm in which internal departures are prioritized at just +/- 5-minute shift in departure errors and prioritize airborne flights in order to reduce the amount of delay that must be absorbed in the air. This, in turn, would push more of the required delay to ground delay for internal departures. Airborne delay is reasonable using the paradigm in which airborne flights are prioritized up to 5-minute error shifts.

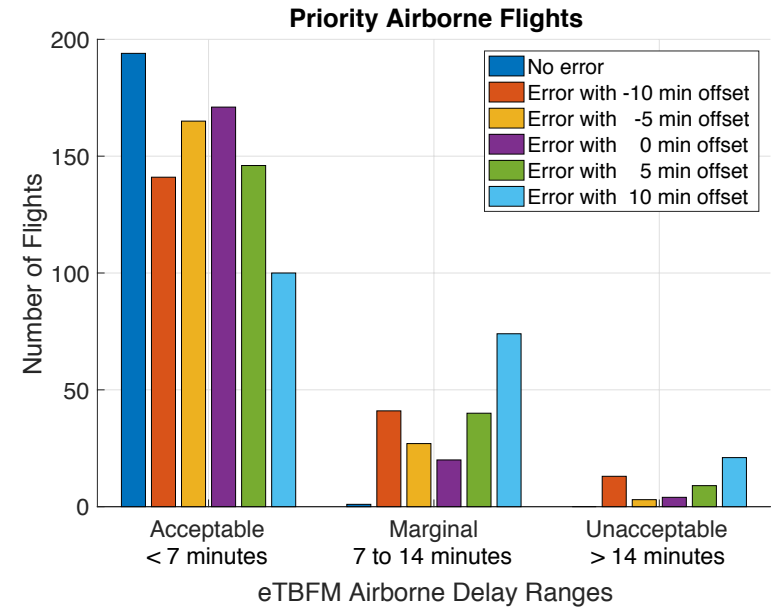

Figure 8 Airborne delay assigned by TBFM using the scheduling paradigm in which airborne flights are priortized. Flights depart with no departure error and fixed depararture error shifted by a variety of offsets.

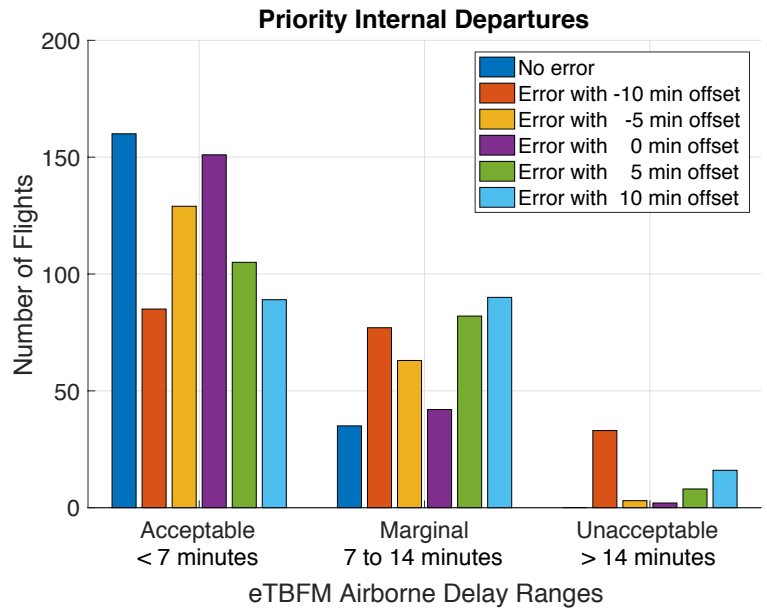

Figure 9 Airborne delay assigned by TBFM using the scheduling paradigm in which internal departures are priortized. Flights depart with no departure error and fixed depararture error shifted by a variety of offsets.

When also considering TBFM ground delay (not shown in the figures), with priority given to airborne flights the total TBFM delays are highest under +10 -minute mean departure error, and lowest under -5 -minute mean departure error. This indicates that, given uncertainty in EDCT compliance, allowing flights to depart before their assigned EDCT could result in lower total TBFM delay. This effect is not present when priority is given to internal departures. In this case, total TBFM delays are lowest under zero mean departure error. This suggests that, with priority given to internal departures, it is important that both early and late departures are minimized, and not only late.

\section{Impact of Departure Error Variability on TBFM Delay Experiment Details}

Gaussian mixture distributions were fitted to the previously generated empirical distributions of departure error, 1,2,3,9 for external flights and internal departures separately. Two-component Gaussian mixtures were used rather than simple Gaussian distributions because the mixture distributions were found to better fit the empirical distributions.

Experiments were conducted to explore the impact of departure error variability, in the form of standard deviation in departure error, on TBFM delay. The departure error distributions for external and internal departures that were derived from the empirical data were modified to simulate improvement and degradation in the conformance to EDCT and call-for-release compliance to study the impact on TBFM assigned delay. Figure 10 and Figure 11 show these adjusted distributions. These distributions were generated by multiplying the standard deviations of the original distributions, represented by $\sigma_{0}$, by $0.5,1.0,2.0,3.0$ and 4.0. For these distributions, the percentage of external flights departing with errors outside \pm 5 minutes is $31 \%, 48 \%, 65 \%, 74 \%$ and $80 \%$, respectively. The percentage of internal flights departing with errors outside -2 to +1 minutes is $9 \%, 30 \%, 58 \%, 72 \%$ and $78 \%$, respectively. When samples were drawn, they were drawn for external and internal departures separately, but from the corresponding distribution (e.g., if external departure errors were sampled from " $3.0 \sigma 0$ ", internal departure errors were sampled from "3.0 $\sigma_{0}$ ").

Simulations were performed using departure errors sampled from each of these distributions for external and internal departures, respectively. When sampling from these distributions to generate departure errors for the various 
experiments, departure errors greater than 60 minutes were discarded and a new error was sampled from the distribution. Five sample sets were drawn from each of the five distribution pairs, for a total of 25 simulation runs. Additional departure errors were generated by increasing these sampled departure errors by 5 minutes, for a further 25 simulation runs with a 5 -minute offset and a total of 50 experiments.

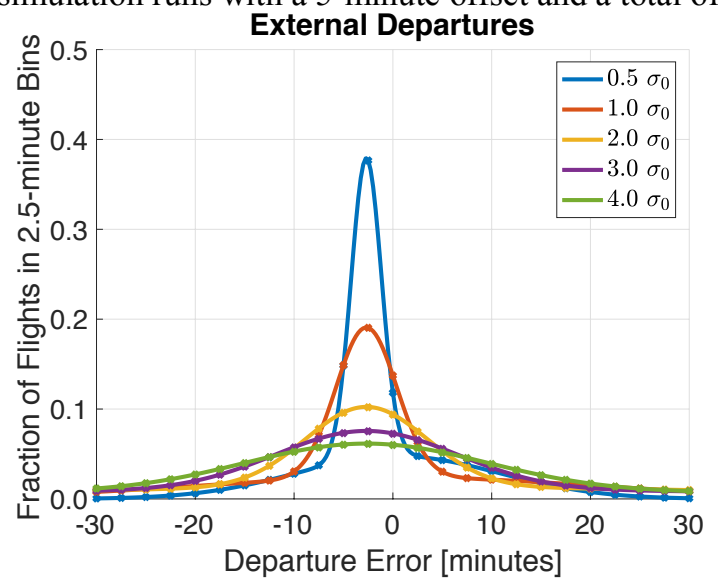

Figure 10. Departure error distributions for external departures modified from the distribution fit to empirical data.

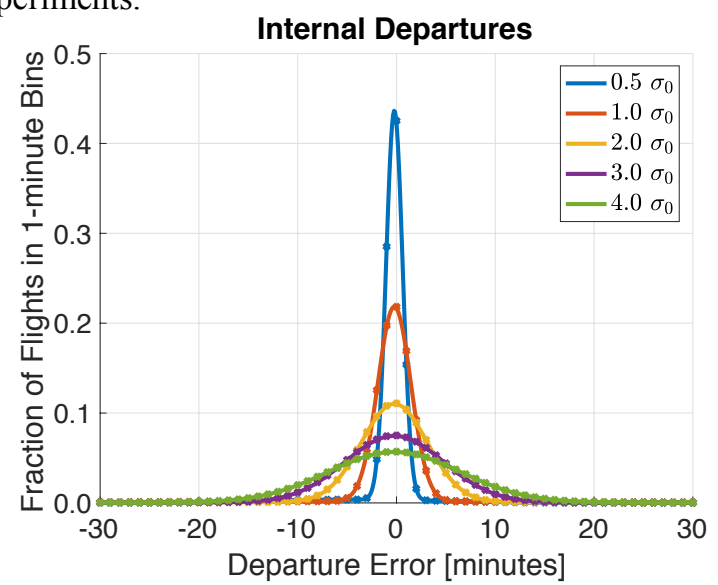

Figure 11. Departure error distributions for internal departures modified from the distribution fit to empirical data.

\section{Results}

Both TBFM scheduling paradigms (i.e., prioritizing airborne flights and prioritizing internal departures) were applied in post-processing on all thirty of the simulation runs from MACS. Results for zero-offset departure error are shown in Figure 12 and Figure 13, with corresponding results for departure error offset by 5 minutes shown in Figure 14 and Figure 15. Figure 12 and Figure 14 show results for the paradigm in which priority is given to airborne flights, while Figure 13 and Figure 15 show results for the paradigm in which priority is given to internal departures.

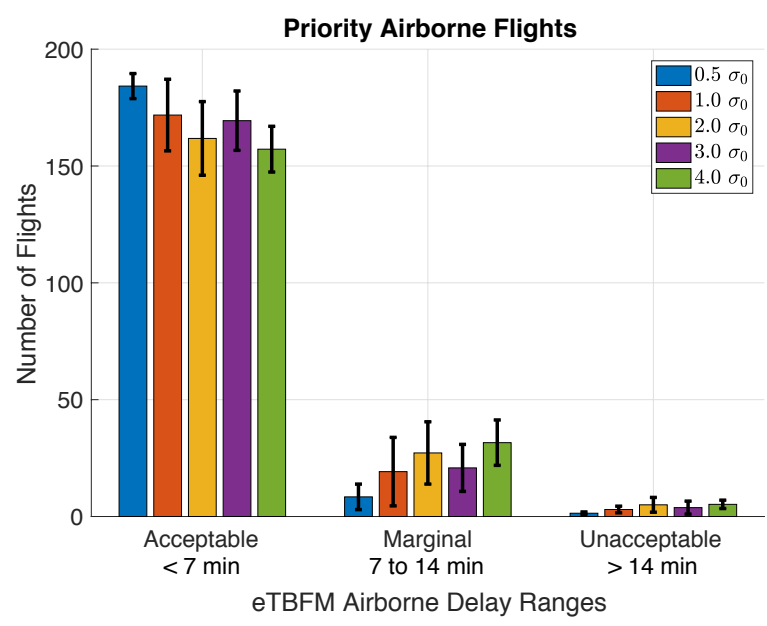

Figure 12. Airborne delay assigned by TBFM using the scheduling paradigm in which airborne flights are priortized. Flights departed with error sampled from distributions given in Figure 10 and Figure 11, and means of 0 minutes.

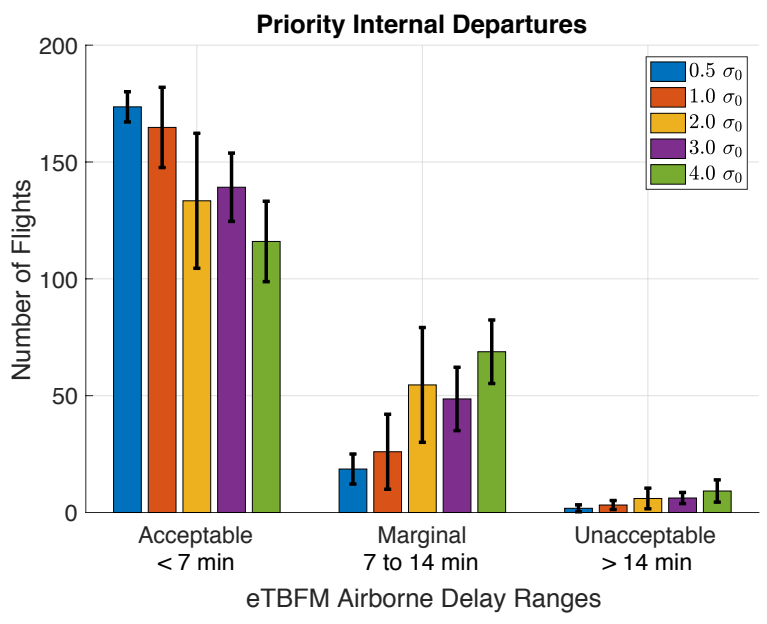

Figure 13. Airborne delay assigned by TBFM using the scheduling paradigm in which internal departures are priortized. Flights departed with error sampled from distributions given in Figure 10 and Figure 11, and means of 0 minutes.

Looking at TBFM airborne delay for the case in which departure error offset is zero minutes (Figure 12 and Figure 13) there is a general trend of delay shifting towards marginal values as the standard deviation of the departure error increases - the number of flights with acceptable delay decreases, while the number with marginal delay increases for $2.0 \sigma_{0}, 3.0 \sigma_{0}$ and $4.0 \sigma_{0}$ over the relatively lower number of flights given marginal delay at $0.5 \sigma_{0}$ and $1.0 \sigma_{0}$. This is true both for the scheduling paradigm in which airborne flights are prioritized (Figure 12) and in the scheduling 
paradigm which internal departures are prioritized (Figure 13). In the paradigm in which airborne flights are prioritized (Figure 12), relatively few aircraft are assigned marginal delay -31.6 flights on average for a standard deviation in departure error of $4.0 \sigma_{0}$. However, in the case in which internal departures are prioritized (Figure 13), the marginal delays affect 68.8 flights on average for a standard deviation in departure error of $4.0 \sigma_{0}$. Under the scheduling paradigm in which airborne flights are prioritized (Figure 12), the number of flights assigned marginal delay stays relatively constant with changes in error variability. This is because required delay is absorbed by internal departures on the ground. Under the scheduling paradigm in which internal departures are prioritized (Figure 13), the increased number of flights assigned marginal delay is more pronounced. This suggests that, in the conditions simulated, high variability in departure error may indeed limit the feasibility of prioritizing internal departure scheduling in TBFM.

When departure errors are increased by a constant 5-minute offset (Figure 14 and Figure 15), the number of flights with acceptable delay, across all runs, decreases relative to the 0-departure error offset results (Figure 12 and Figure 13). This trend is also shown in Figure 6 and Figure 7 above. Correspondingly, the number of flights with marginal and unacceptable delay increase, across all runs.

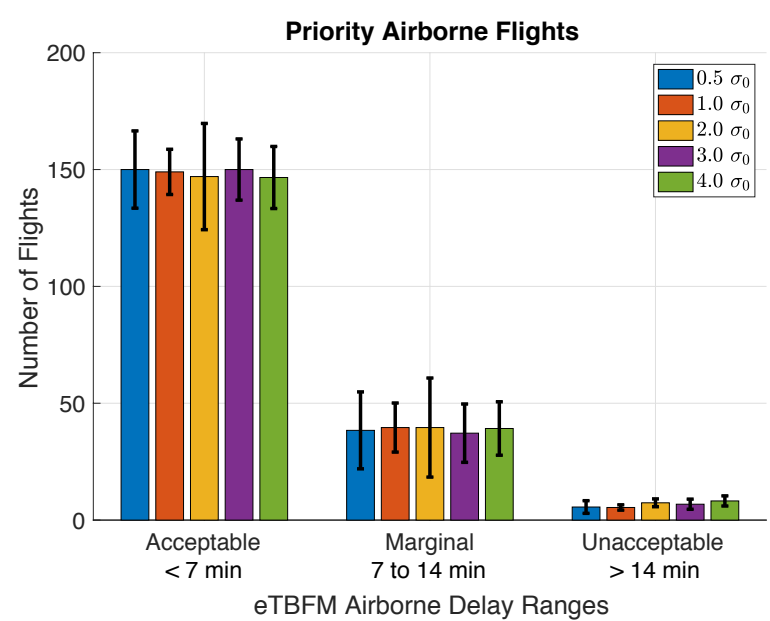

Figure 14. Airborne delay assigned by TBFM using the scheduling paradigm in which airborne flights are priortized. Flights departed with error sampled from distributions given in Figure 10 and Figure 11, and offset by 5 minutes.

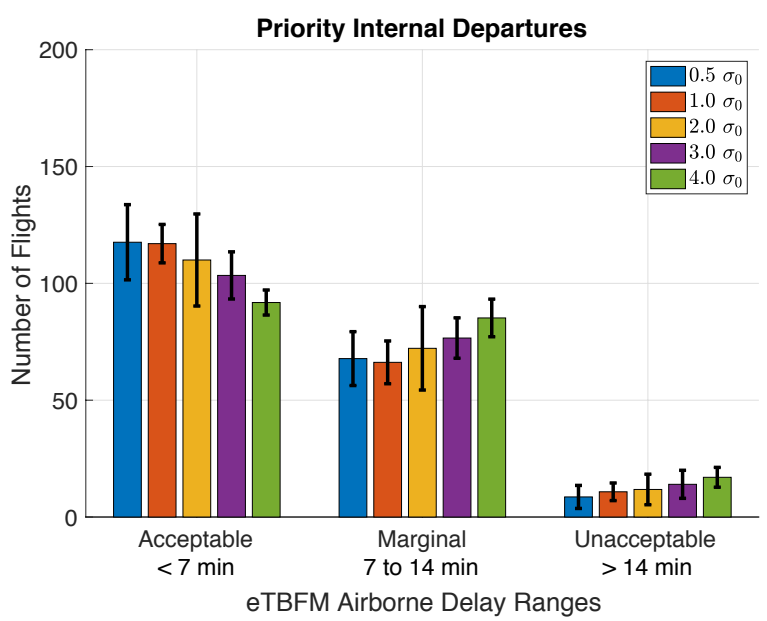

Figure 15. Airborne delay assigned by TBFM using the scheduling paradigm in which internal departures are priortized. Flights departed with error sampled from distributions in Figure 10 and Figure 11, and offset by 5 minutes.

When airborne flights are prioritized the number of flights incurring acceptable, marginal and unacceptable delay, respectively, remains relatively constant over variations in the standard deviation of departure error. However, when internal departures are prioritized, the number of flights incurring acceptable delay decreases as the departure error standard deviation increases. The total decrease in the number of flights given acceptable delay is 25.8, on average, as the variability in departure error increases from $0.5 \sigma_{0}$ to $4.0 \sigma_{0}$. There is a corresponding increase in flights with both marginal (by 17.4 flights) and unacceptable (by 8.4 flights) delay. These results further suggest that, in the conditions simulated, high variability in departure error may indeed limit the feasibility of prioritizing internal departure scheduling in TBFM. However, comparing to the results in Figure 12 and Figure 13, these results suggest that the impact of offset errors on airborne delay may dominate over the impact of error variability.

\section{Conclusions and Future Work}

While there is a wide range of parametric studies that could be conducted in order to gain greater insight into the management of demand under capacity constrained resources using TMIs, two of them were addressed here, namely: one to quantify the impact of varying levels of airline participation in the user negotiation of route options, presented in Section IV, and a second to quantify the impact of departure uncertainty on tactical TBFM delays, presented in Section V. TMIAutoSim, a simulation tool developed to support parametric studies and existing HITL capabilities, was used to conduct these studies. The airline participation study may help incentivize airlines to participate in CTOP. The results of the departure error studies can be used to either support EDCT and call-for-release conformance ranges or be used to determine when to switch between TBFM scheduling paradigms that prioritize internal departures or airborne flights. 
Experimental results suggest that, for the scenario studied, as CTOP participation increases in the form of airlines submitting TOSs with multiple trajectory options (as opposed to a single option TOS), average ground delays assigned to aircraft going through the FCA decreases. This supports the findings of Ref. 12 that there are significant benefits to airlines by participating in CTOP. A threshold in CTOP participation, which varies with the constraint capacity, is also identified beyond which there is relatively little further reduction in average ground delays. Given the likely level of CTOP participation, the capacity reduction for which CTOP would be an appropriate TMI, resulting in acceptable levels of delay, is also identified.

Experimental results from the paper suggest that high average departure errors (either positive or negative), and high variability in departure error, can make the prioritization of internal departures in TBFM departure metering and scheduling infeasible. Results presented here show that average departure errors of $+/-10$ minutes causes marginal airborne delay for 31.6 and 68.8 flights, respectively, when internal departures are prioritized. When departure errors of these magnitudes are observed, a switch from prioritizing internal departures to prioritizing airborne flights would decrease unacceptable airborne delay. Alternatively, these results could be used to justify requiring average departure delays to be within $+/-5$ minutes to ensure the feasibility of TBFM scheduling which prioritized internal departures.

Further work will extend the capabilities of TMIAutoSim. This will include, particularly, (1) to simulate the absorption of TBFM airborne delay using speed control, and (2) to simulate TBFM extended metering, which includes multiple uncoupled schedules for each flow, to a series of metering points, each with its own freeze horizon. Extended metering is another capability that forms a key component of the IDM concept, and is applied in the ongoing HITL simulations supporting IDM. Automatic simulation of TBFM airborne delay absorption would allow aircraft to meet scheduled times of arrival specified by TBFM, which is required for simulating extended metering, or other constraints, such as meet miles-in-trail requirements. The latter would allow for the simulation of current day operations, allowing benefits of the IDM concept to be quantified across a range of traffic and capacity scenarios. TMIAutoSim will also be developed to enable fast-time simulation, which would contribute to studying a wider range of parameters than is currently possible in real-time.

\section{References}

${ }^{1}$ Smith, N., Brasil, C., Lee, P.U., Buckley, N., Gabriel, C., Mohlenbrink., C, Omar, F., Parke., B., Speridakos, C., and Yoo, H., Integrated Demand Management: Coordinating Strategic and Tactical Flow Scheduling Operations, 16th AIAA Aviation Technology, Integration, and Operations Conference, 2016, AIAA 2016-4221.

${ }^{2}$ Yoo, H.S., Brasil, C., Buckley, N., Mohlenbrink, C., Speridakos, C., Parke, B., Hodell, G., Lee, P.U., and Smith, N.M., Integrated Demand Management: Minimizing Unanticipated Excessive Departure Delay while Ensuring Fairness from a Traffic Management Initiative, 17th AIAA Aviation Technology, Integration, and Operations Conference, 2017, AIAA $2017-4100$.

${ }^{3}$ Arneson, H., Evans, A. D., Li, J., Wei, M.Y., Development and validation of an automated simulation capability in support of Integrated Demand Management, Royal Aeronautical Society Flight Simulation Conference, November 2017, RAeS, AIAA.

${ }^{4}$ P. Smith, E. Stellings, "Operating in a CTOP (Collaborative Trajectory Options Program) Environment", CDM Flow Evaluation Team, Tech. Rep., 2014.

5 “TFMS Functional Description, Appendix C: Traffic Management Initiative (TMI) Algorithms”, CSC/TFMM-13/1744, Tech. Rep., 2014.

${ }^{6}$ Tyo, R., and Pressler, C., Industry Day Briefing: Time-Based Flow Management (TBFM), Federal Aviation Administration Industry Day, April, 2014. from:

http://www.faa.gov/about/office_org/headquarters_offices/ato/service_units/pmo/industry day/media/day 1 - tbfm bob tyo chris pressler - final.pdf

[Retrieved June 12, 2017].

${ }^{7}$ Zelinski, S., Xue, M., and Bassett, P., Optimized Route Capability (ORC) Intelligent Offloading of Congested Arrival Routes, 16th AIAA Aviation Technology, Integration, and Operations Conference, 2016, AIAA 2016-4357.

${ }^{8}$ Prevot, T., Exploring the many perspectives of distributed air traffic management: The Multi Aircraft Control System MACS, In Proceedings of the HCI-Aero, 2002, pp. 149-154.

${ }^{9}$ Hyo-Sang Yoo, Connie L. Brasil, Nathan Buckley, Gita S. Hodell, Scott N. Kalush, Paul U. Lee, Nancy M. Smith. "Impact of Different Trajectory Option Set Participation Levels within an Air Traffic Management Collaborative Trajectory Option Program." In 18th AIAA Aviation Technology, Integration, and Operations Conference.

10 Conde Rocha Murca, M., DeLaura, R., Hansman, R.J., Jordan, R., Reynolds, T. and Balakrishnan, H., 2016. Trajectory clustering and classification for characterization of air traffic flows. In 16th AIAA Aviation Technology, Integration, and Operations Conference (p. 3760).

${ }^{11}$ Bombelli, A., Segarra Torne, A., Trumbauer, E. and Mease, K.D., 2017. Automated route clustering for air traffic modeling. In AIAA Modeling and Simulation Technologies Conference (p. 1318).

${ }^{12}$ Yoo, H.S., Mohlenbrink, C., Brasil, C., Buckley, N., Globus, A., Smith, N.M. and Lee, P.U., Required time of arrival as a control mechanism to mitigate uncertainty in arrival traffic demand management, $35^{\text {th }}$ IEEE/AIAA Digital Avionics Systems Conference, 2016, IEEE. 